\title{
SLC19A3 Loss-of-Function Variant in Yorkshire Terriers with Leigh-Like Subacute Necrotizing Encephalopathy
}

\author{
Michaela Drögemüller ${ }^{1, \dagger}$, Anna Letko ${ }^{1,+}{ }^{+}$, Kaspar Matiasek ${ }^{2, \dagger}$, Vidhya Jagannathan ${ }^{1}{ }^{10}$, \\ Daniele Corlazzoli ${ }^{3}$, Marco Rosati ${ }^{2}$, Konrad Jurina ${ }^{4}$, Susanne Medl ${ }^{5}$, Thomas Gödde ${ }^{6}$, \\ Stefan Rupp ${ }^{7}$, Andrea Fischer ${ }^{8}$, Alejandro Luján Feliu-Pascual ${ }^{9}$ (i) and Cord Drögemüller ${ }^{1, *(B)}$ \\ 1 Institute of Genetics, Vetsuisse Faculty, University of Bern, 3012 Bern, Switzerland; \\ michaela.droegemueller@vetsuisse.unibe.ch (M.D.); anna.letko@vetsuisse.unibe.ch (A.L.); \\ vidhya.jagannathan@vetsuisse.unibe.ch (V.J.) \\ 2 Section of Clinical \& Comparative Neuropathology, Centre for Clinical Veterinary Medicine, Ludwig \\ Maximilians Universität Munich, 80539 Munich, Germany; kaspar.matiasek@neuropathologie.de (K.M.); \\ marco.rosati@neuropathologie.de (M.R.) \\ 3 Neurology \& Neurosurgery Unit, Policlinico Veterinario Roma Sud, 00173 Roma, Italy; \\ daniele.corlazzoli@me.com \\ 4 Small Animal Hospital, Tierklinik Haar, 85540 Haar, Germany; datenschutz.haar@anicura.de \\ 5 Small Animal Hospital, Anicura Kleintierklinik Babenhausen, 87727 Babenhausen, Germany; \\ smedl@tierklinik-medl.de \\ 6 Small Animal Referral Practice, Veterinary Health Centre, 83451 Piding, Germany; \\ t.goedde@tierarzt-piding.com \\ 7 Small Animal Hospital, Tierklinik Hofheim, 65719 Hofheim, Germany; s.rupp@tierklinik-hofheim.de \\ 8 Section of Neurology, Centre for Clinical Veterinary Medicine, Ludwig-Maximilians-Universität, \\ 80539 Munich, Germany; a.fischer@medizinische-kleintierklinik.de \\ 9 Aúna Especialidades Veterinarias, 46980 Valencia, Spain; alf@aunaespecialidadesveterinarias.es \\ * Correspondence: cord.droegemueller@vetsuisse.unibe.ch \\ + These authors contributed equally to the work.
}

Received: 17 September 2020; Accepted: 15 October 2020; Published: 16 October 2020

\begin{abstract}
Sporadic occurrence of juvenile-onset necrotizing encephalopathy (SNE) has been previously reported in Yorkshire terriers. However, so far, no causative genetic variant has been found for this breed-specific form of suspected mitochondrial encephalomyopathy. Affected dogs showed gait abnormalities, central visual defects, and/or seizures. Histopathological analysis revealed the presence of major characteristics of human Leigh syndrome and SNE in Alaskan huskies. The aim of this study was to characterize the genetic etiology of SNE-affected purebred Yorkshire terriers. After SNP genotyping and subsequent homozygosity mapping, we identified a single loss-of-function variant by whole-genome sequencing in the canine SLC19A3 gene situated in a $1.7 \mathrm{Mb}$ region of homozygosity on chromosome 25 . All ten cases were homozygous carriers of a mutant allele, an indel variant in exon 2, that is predicted to lead to a frameshift and to truncate about $86 \%$ of the wild type coding sequence. This study reports a most likely pathogenic variant in SLC19A3 causing a form of SNE in Yorkshire terriers and enables selection against this fatal neurodegenerative recessive disorder. This is the second report of a pathogenic alteration of the SLC19A3 gene in dogs with SNE.
\end{abstract}

Keywords: Canis familiaris; whole-genome sequencing; rare disease; precision medicine; neurometabolic disorder 


\section{Introduction}

Subacute necrotizing encephalomyelopathy (SNE), also termed Leigh syndrome (LS; OMIM 256000) represents a devastating neurodegenerative disorder in people, characterized by a wide variety of clinical signs, ranging from severe neurologic problems to a near absence of abnormalities with the central nervous system most frequently affected [1]. Originally, Archibald Denis Leigh, a British neuropsychiatrist described the condition in 1951 [2]. SNE is characterized by focal and bilaterally symmetrical, necrotic lesions involving the thalamus, brainstem, and posterior columns of the spinal cord [3]. In SNE, various mutations in mitochondrial respiratory chain complexes lead to the disruption of ATP synthesis resulting in the characteristic pathology of SNE [4]. Mitochondrial encephalomyelopathies, such as SNE or LS, represent rare inherited neurometabolic disorders showing considerable genetic heterogeneity and associated pathogenic variants affecting over 85 different genes of the mitochondrial or nuclear genome [3]. Therefore, they represent mitochondrial disorders with the largest genetic heterogeneity [1].

As human SNE is rare and heterogeneous, studying domestic animal species showing resembling conditions might add to the understanding of such a complex group of disorders. Rare forms of SNE were described e.g., in cattle [5-7] and dogs (OMIA 001097-9615). The first report of this disorder was described in Alaskan huskies [8,9] and subsequently, a similar form of SNE was reported in Yorkshire terriers [10] and American Staffordshire bull terriers [11]. Neuropathologically, SNE in Yorkshire terriers is nearly identical to the Alaskan husky form and very similar to human Leigh syndrome [10]. An initial genetic investigation of SNE-affected Yorkshire terriers revealed no indication for disease-causing variants in the mitochondrial genome [10], whereas more recently in Alaskan huskies the pathogenesis of recessively inherited SNE was unraveled [12,13]. This breed-specific fatal brain disorder in Alaskan huskies is associated with a deleterious loss-of-function variant in SLC19A3 encoding for a thiamine transporter 2 (THTR2) with a predominately central nervous system (CNS) distribution $[12,13]$. The SLC19A3 gene product controls the uptake of thiamine in the CNS via expression of the thiamine transporter protein THTR2. Pathogenic variants are associated with thiamine metabolism dysfunction syndrome-2 in people (THMD2; OMIM 607483), also known as biotin-responsive basal ganglia disease (BBGD) or thiamine-responsive encephalopathy [14]. This SLC19A3-related condition is an autosomal recessive disorder with childhood-onset that presents as a subacute encephalopathy and progresses to severe cogwheel rigidity, dystonia, quadriparesis, and eventually death if left untreated (OMIM 606152). The SLC19A3-related SNE of Alaskan huskies was proposed as a possible large animal model that may allow prospective investigations into the mechanisms of SLC19A3-related syndromes and the potential role of thiamine and/or biotin as a therapeutic strategy $[12,13]$.

To elucidate the disease mechanism underlying monogenic autosomal recessive inherited SNE in Yorkshire terriers, we applied homozygosity mapping and whole-genome sequencing revealing a most likely pathogenic variant in the canine SLC19A3 gene.

\section{Materials and Methods}

\subsection{Ethics Statement}

All animal experiments were performed according to the local regulations. All animals in this study were examined with the consent of their owners. Sample collection was approved by the Cantonal Committee for Animal Experiments (Canton of Bern; permit 71/19).

\subsection{Animals}

In total, 172 blood samples of Yorkshire terriers were collected. Ten dogs were diagnosed with Leigh-like subacute necrotizing encephalopathy (SNE) according to Baiker et al. [10]. These affected dogs were unrelated, apart from two full siblings for which their sire and dam (obligate carriers) as well as a single normal littermate were also available. The remaining 159 dogs represented unrelated 
purebred controls. Genomic DNA was isolated from EDTA blood samples using the Maxwell RSC Whole Blood DNA kit (Promega, Dübendorf, Switzerland).

\subsection{Single Nucleotide Polymorphism Array Genotyping}

Four selected SNE-affected Yorkshire terriers were genotyped on Illumina CanineHD BeadChip array (Illumina, San Diego, CA, USA). PLINK v1.9 [15] was used to perform the quality control filtering steps of the obtained genotyping data and the subsequent homozygosity analysis. Single nucleotide polymorphisms (SNP) with a call rate $<90 \%$ were removed leaving 167,185 markers. All individuals had call rates $>90 \%$. Homozygosity analysis was carried out with PLINK v1.9 [15] to determine intervals of extended homozygous regions with alleles shared by all four affected dogs.

\subsection{Whole-Genome Sequencing}

Whole-genome sequence (WGS) data of a single affected dog was obtained at $19.7 \times$ coverage in order to identify the causative variant for SNE. The sequence data analysis and calling of single nucleotide variants and small indels (SNVs) including the prediction of functional effects were described before [16]. The dog reference genome assembly CanFam3.1 and NCBI annotation release 105 was used. Additionally, a publicly available control genomes cohort of 720 dogs from 130 various breeds, and 9 wolves [16] was used to filter variants private in the sequenced SNE-affected dog; this also included 60 unrelated Yorkshire terriers (Supplementary Table S1). The Integrative genomics viewer (IGV) software [17] was used for visual inspection and screening for structural variants in the associated regions.

\subsection{Sanger Sequencing and Targeted Genotyping}

Polymerase chain reaction (PCR) and Sanger sequencing were used to validate and characterize the SLC19A3 indel variant (XM_022409850.1:c.205_210delins35) identified from whole-genome sequencing. PCR primers were designed using primer 3 [18]. PCR products from genomic DNA were amplified using AmpliTaqGold360 MasterMix (Thermo Fisher Scientific, Waltham, MA, USA) and the purified PCR amplicons were directly sequenced on an ABI3730 capillary sequencer (Thermo Fisher Scientific) using the following primers: GGCAGTCACCATCCCATAGA (forward) and GATATTGGGCAAGCCACCTA (reverse) generating 309 bp products. The sequence data were analyzed with Sequencher 5.1 software (GeneCodes, Ann Arbor, MI, USA). Diagnostic genotyping was performed by fragment length analysis using a different forward primer (ATCCCTTGCAGGATGATGAC) to produce amplicons of $218 \mathrm{bp}$ or $247 \mathrm{bp}$ representing the wild type or variant allele, respectively. The $29 \mathrm{bp}$ size difference was visualized on a Fragment Analyzer capillary gel electrophoresis instrument (Advanced Analytical Technologies, Ames, IA, USA).

\subsection{Availability of Data and Material}

The whole-genome data of an SNE-affected Yorkshire terrier are freely available at the European Nucleotide Archive (ENA) under sample accession number SAMEA3928145. All accession numbers of the used control genomes are available in Supplementary Table S1.

All genome positions are reported with respect to dog reference genome assembly CanFam3.1 and NCBI annotation release 105. All references to the canine SLC19A3 gene correspond to the accessions NC_006607.3 (NCBI accession), XM_022409850.1 (mRNA), and XP_022265558.1 (protein).

\section{Results}

\subsection{Homozygosity Analysis}

Based on the clinicopathological diagnosis of Leigh-like subacute necrotizing encephalopathy (SNE) in all examined Yorkshire terriers and the similarities to the recessively inherited conditions in SNE-affected Alaskan husky dogs and THMD2-affected humans, as well as the available pedigree 
information of the two SNE-affected siblings, a recessive mode of inheritance was postulated. Therefore, homozygosity mapping assuming identity-by-descent (IBD) was used to determine critical genomic regions shared across four SNP array genotyped cases. This revealed five genome regions with a total of $\sim 4.1 \mathrm{Mb}$ located on five different dog chromosomes (Table 1), representing $0.17 \%$ of the canine reference sequence. Visual inspection of these regions in the WGS of the affected dog did not reveal any evidence for copy number variants or large structural rearrangements.

Table 1. Regions of shared homozygosity detected in four subacute necrotizing encephalomyelopathy (SNE)-affected Yorkshire terriers.

\begin{tabular}{ccccc}
\hline \multirow{2}{*}{ Chromosome } & \multicolumn{2}{c}{ Position $^{\mathbf{1}}$} & Length (kb) & $\begin{array}{c}\text { Number of Annotated } \\
\text { Protein-Coding Genes in the Region }\end{array}$ \\
& Start & End & & 0 \\
3 & $44,184,889$ & $44,286,148$ & 101.3 & 1 \\
6 & $71,329,720$ & $71,552,171$ & 222.5 & 22 \\
10 & $20,608,121$ & $22,376,735$ & 1768.6 & 13 \\
25 & $39,477,619$ & $41,191,570$ & 1714.0 & 2 \\
31 & $33,337,422$ & $33,591,249$ & 253.8 & \\
\hline \multicolumn{6}{c}{} \\
\hline \multicolumn{7}{c}{ in respect to dog reference genome assembly CanFam3.1. }
\end{tabular}

\subsection{Identification of the Causative Variant}

Filtering the variants of a single affected Yorkshire terrier against 729 public control genomes [16], including 60 breed controls, for single-nucleotide variants (SNVs) and short indels present in the five identified IBD-regions resulted in only a single private protein-changing variant (Figure 1a). The indel affecting $\sim 45 \mathrm{bp}$ is located in exon 2 of the thiamine transporter 2 (LOC486151) gene, also known as solute carrier family 19 members 3 (SLC19A3) gene (Figure 1b). PCR and subsequent Sanger sequencing confirmed the homozygous presence of this small structural variant in SNE-affected Yorkshire terriers and revealed the detailed features of the indel: a $35 \mathrm{bp}$ insertion replacing $6 \mathrm{bp}$ and thereby disturbing the correct reading frame (Figure 1c). There are 15 currently annotated transcript isoforms for the canine SLC19A3, which is in reverse complementary orientation with respect to the canine reference genome. While the canine SLC19A3 protein length is 495 amino acids, the human protein (NP_001358340.1) has 496 amino acids, from which $408(82.3 \%)$ are identical between dog and human. The Yorkshire terrier variant leads to a frameshift and a premature stop codon (c.205_210delins35; p.Pro69Ilefs*45) truncating $\sim 86 \%$ of the wild type coding sequence (Figure 1c). 
a
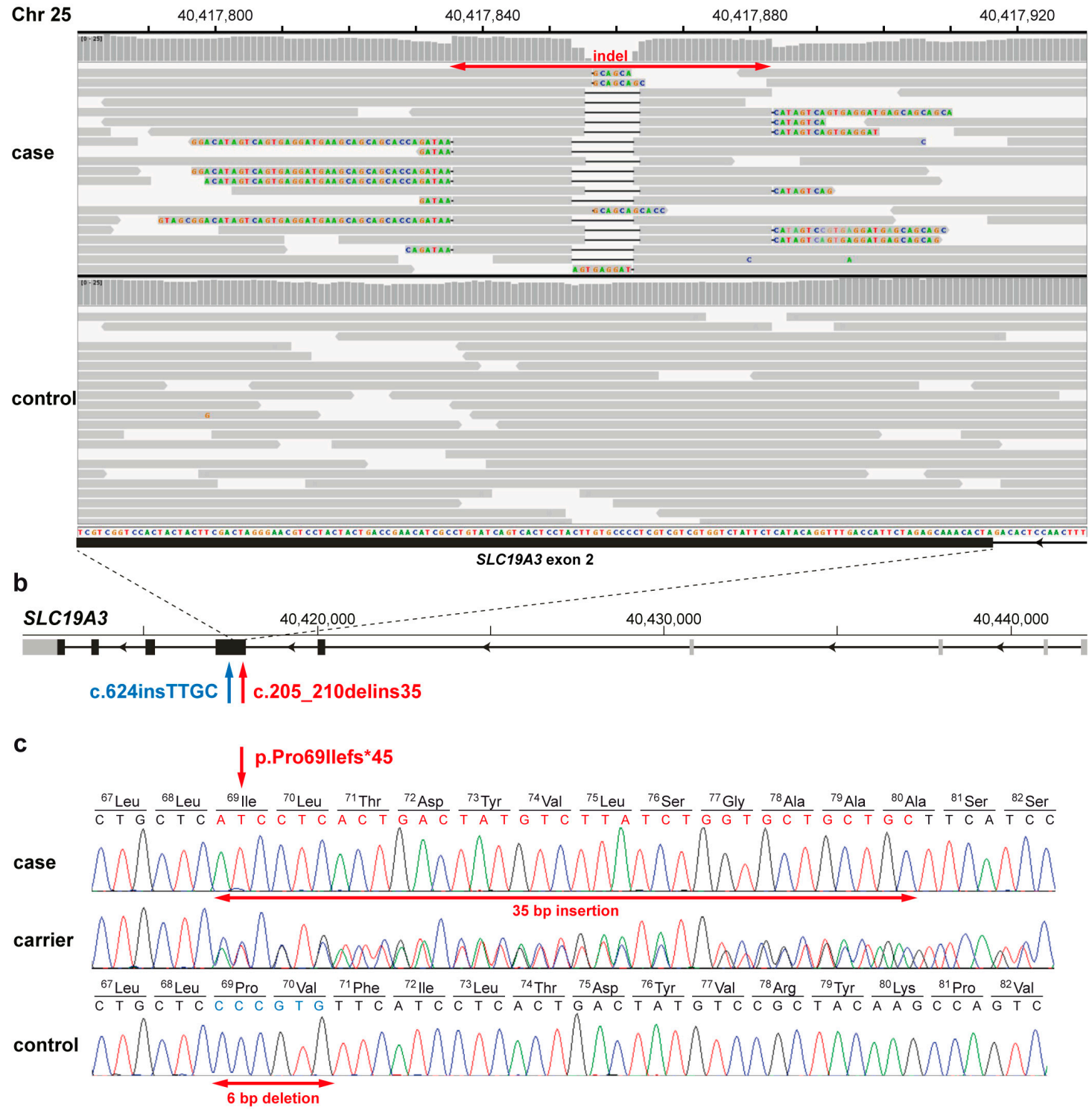

Figure 1. Subacute necrotizing encephalopathy (SNE)-associated SLC19A3 loss-of-function variant in Yorkshire terriers. (a) IGV [17] screenshots of the genome region on canine chromosome 25 with the SLC19A3:c.205_210delins35 variant in an affected and a control Yorkshire terrier (NC_006607.3:40417780-40417930); The indel variant detected in the SNE-affected dog is indicated by a red arrow. (b) Schematic representation of the canine SLC19A3 gene showing the location of both pathogenic variants in exon 2 (XM_022409850.1): the herein identified indel (red arrow) and the insertion previously described in encephalopathy-affected Alaskan huskies (blue arrow) [12]. Note that the number of $5^{\prime}$-untranslated exons (grey) varies between transcript isoforms, whereas the five protein-coding $3^{\prime}$-exons (black) are more conserved; (c) Sanger sequencing electropherograms illustrate sequences of a homozygous SNE-affected Yorkshire terrier, a heterozygous carrier, and a homozygous wild type dog. The red arrows indicate that the 35 bases shown in red are inserted, whereas the 6 bases in blue are deleted in the mutant allele. The predicted consequence of the shift in the reading frame altering the amino acid sequence of the SLC19A3 protein and leading to a premature stop is shown above.

\subsection{Targeted Genotyping of the Variant}

Genotyping by fragment size analysis of the 172 available Yorkshire terriers confirmed perfect segregation of the detected SLC19A3 variant with the observed disease phenotype. Only the ten 
SNE-affected dogs were homozygous for the variant allele (Table 2). Two obligate carriers and one tested normal littermate were heterozygous carriers of the variant, while 162 controls tested homozygous for the wild type allele (Table 2).

Table 2. Segregation of the SLC19A3: c.205_210delins35 genotypes with subacute necrotizing encephalopathy in Yorkshire terriers.

\begin{tabular}{cccc}
\hline SNE Status & wt/wt & wt/var & var/var \\
\hline Affected $(n=10)$ & 0 & 0 & 10 \\
Non-affected $(n=222){ }^{1}$ & 219 & $3^{2}$ & 0 \\
\hline
\end{tabular}

1 including 60 dogs with WGS data ${ }^{2}$ includes 2 obligate carriers and 1 normal littermate of the affected dogs.

\section{Discussion}

In this study, the obtained genetic results elucidate the underlying aetiology of the previous clinical and pathological characterization of a Leigh-like subacute necrotizing encephalopathy in the affected Yorkshire terriers, which resembles the human Leigh syndrome. The SLC19A3 variant found by a combination of SNP genotyping-based homozygosity mapping and whole-genome sequencing, confirmed by Sanger sequencing, segregated perfectly in the investigated cohort of $>200$ unrelated Yorkshire terriers.

Numerous homozygous as well as compound heterozygous variants have been reported before in different regions of SLC19A3 in human patients suffering from thiamine metabolism dysfunction syndrome-2 [19]. SLC19A3 is a member of solute carrier family 19 and encodes thiamine transporter 2. Together with thiamine transporter 1 , it is necessary for transport and homeostasis of thiamine that is important in brain development. [20]. Slc19a3-knockout mice showed progressive wasting and lethargy leading to a premature death as well as a significant decrease in thiamin uptake, even though there were no obvious histological changes in the brain [21].

The herein-described most likely pathogenic variant (XP_022265558.1:p.Pro69Ilefs*45) lies within the second of 12 transmembrane domains of the SLC19A3 protein and, therefore, affects $\sim 86 \%$ of the wild type sequence. The SLC19A3 gene probability of loss-of-function intolerance is pLI $=0.104$ [22], which indicates variants in SLC19A3 leading to a loss of gene function are most likely recessive, where loss of a single copy is often tolerated but the loss of both copies is not. The herein-described variant leads to an insertion of a premature termination in the second out of five coding exons, suggesting that any synthesized mRNA would likely be degraded through nonsense-mediated decay, unlikely to produce a fully functional protein. Heterozygous carriers did not show a visible clinical phenotype, as they can most likely compensate due to the presence of the normal protein, albeit at a decreased amount.

In conclusion, our results provide strong evidence for a breed-specific deleterious variant in SLC19A3 as the most likely genetic cause of monogenic autosomal recessive Leigh-like subacute necrotizing encephalopathy in Yorkshire terriers, and they enable the development of a genetic test for veterinary diagnostic and breeding decisions. Finally, this presents the second, most likely breed-specific pathogenic variant in the canine SLC19A3 gene in SNE-affected dogs.

Supplementary Materials: The following are available online at http://www.mdpi.com/2073-4425/11/10/1215/s1, Table S1: Accession numbers of $721 \mathrm{dog}$ and 9 wolf whole-genome genome sequences.

Author Contributions: Conceptualization, K.M. and C.D.; methodology, M.D.; data curation, V.J.; formal analysis, M.D. and A.L.; investigation, M.D.; resources, K.M., D.C., M.R., K.J., S.M., T.G., S.R., A.F. and A.L.F.-P.; writing-original draft preparation, A.L., M.D., K.M. and C.D.; writing—review and editing, M.D., A.L., K.M. and C.D.; visualization, A.L. and C.D.; software, V.J.; supervision, C.D.; funding acquisition, C.D. All authors have read and agreed to the published version of the manuscript.

Funding: This research received no external funding.

Acknowledgments: The authors are grateful to the owners of all dogs who provided samples and shared valuable information. The Next Generation Sequencing Platform and the Interfaculty Bioinformatics Unit of the University of Bern are acknowledged for performing the WGS and providing high-performance computational infrastructure. 
Conflicts of Interest: The authors declare no conflict of interest.

\section{References}

1. Finsterer, J. Leigh and Leigh-Like Syndrome in Children and Adults. Pediatr. Neurol. 2008, 39, $223-235$. [CrossRef] [PubMed]

2. Leigh, D. Subacute necrotizing encephalomyelopathy in an infant. J. Neurol. Neurosurg. Psychiatry 1951, 14, 216-221. [CrossRef] [PubMed]

3. Chang, X.; Wu, Y.; Zhou, J.; Meng, H.; Zhang, W.; Guo, J. A meta-analysis and systematic review of Leigh syndrome: Clinical manifestations, respiratory chain enzyme complex deficiency, and gene mutations. Medicine 2020, 99, e18634. [CrossRef] [PubMed]

4. Fecek, C.; Samanta, D. Subacute Necrotizing Encephalomyelopathy (Leigh Syndrome). Available online: http://www.ncbi.nlm.nih.gov/pubmed/32644590 (accessed on 14 September 2020).

5. Steffen, D.J.; Vestweber, J.G.; Cash, W.; El-Hamidi, M.; Leipold, H.W. Multifocal Subacute Necrotizing Encephalomyelopathy in Simmental Calves. J. Vet. Diagn. Investig. 1994, 6, 466-472. [CrossRef]

6. Desjardins, I.; Fecteau, G.; Hélie, P.; Desrochers, A. Multifocal subacute necrotizing encephalomyelopathy in a Simmental calf. Can. Vet. J. 2001, 42, 375-377.

7. Philbey, A.W.; Martel, K.S. A multifocal symmetrical necrotising encephalomyelopathy in Angus calves. Aust. Vet. J. 2003, 81, 226-229. [CrossRef] [PubMed]

8. Wakshlag, J.J.; de Lahunta, A.; Robinson, T.; Cooper, B.J.; Brenner, O.; O’Toole, T.D.; Olson, J.; Beckman, K.B.; Glass, E.; Reynolds, A.J. Subacute necrotising encephalopathy in an Alaskan husky. J. Small Anim. Pract. 1999, 40, 585-589. [CrossRef]

9. Brenner, O.; Wakshlag, J.J.; Summers, B.A.; de Lahunta, A. Alaskan Husky encephalopathy-A canine neurodegenerative disorder resembling subacute necrotizing encephalomyelopathy (Leigh syndrome). Acta Neuropathol. 2000, 100, 50-62. [CrossRef] [PubMed]

10. Baiker, K.; Hofmann, S.; Fischer, A.; Gödde, T.; Medl, S.; Schmahl, W.; Bauer, M.F.; Matiasek, K. Leigh-like subacute necrotising encephalopathy in Yorkshire Terriers: Neuropathological characterisation, respiratory chain activities and mitochondrial DNA. Acta Neuropathol. 2009, 118, 697-709. [CrossRef] [PubMed]

11. Collins, D.; Angles, J.M.; Christodoulou, J.; Spielman, D.; Lindsay, S.A.; Boyd, J.; Krockenberger, M.B. Severe Subacute Necrotizing Encephalopathy (Leigh-like Syndrome) in American Staffordshire Bull Terrier Dogs. J. Comp. Pathol. 2013, 148, 345-353. [CrossRef] [PubMed]

12. Vernau, K.M.; Runstadler, J.A.; Brown, E.A.; Cameron, J.M.; Huson, H.J.; Higgins, R.J.; Ackerley, C.; Sturges, B.K.; Dickinson, P.J.; Puschner, B.; et al. Genome-Wide Association Analysis Identifies a Mutation in the Thiamine Transporter 2 (SLC19A3) Gene Associated with Alaskan Husky Encephalopathy. PLoS ONE 2013, 8, e57195. [CrossRef] [PubMed]

13. Vernau, K.; Napoli, E.; Wong, S.; Ross-Inta, C.; Cameron, J.; Bannasch, D.; Bollen, A.; Dickinson, P.; Giulivi, C. Thiamine Deficiency-Mediated Brain Mitochondrial Pathology in Alaskan Huskies with Mutation in SLC19A3.1. Brain Pathol. 2015, 25, 441-453. [CrossRef] [PubMed]

14. Marcé-Grau, A.; Martí-Sánchez, L.; Baide-Mairena, H.; Ortigoza-Escobar, J.D.; Pérez-Dueñas, B. Genetic defects of thiamine transport and metabolism: A review of clinical phenotypes, genetics and functional studies. J. Inherit. Metab. Dis. 2019, 42, 581-597. [CrossRef] [PubMed]

15. Chang, C.C.; Chow, C.C.; Tellier, L.C.A.M.; Vattikuti, S.; Purcell, S.M.; Lee, J.J. Second-generation PLINK: Rising to the challenge of larger and richer datasets. Gigascience 2015, 4, 1-16. [CrossRef] [PubMed]

16. Jagannathan, V.; Drögemüller, C.; Leeb, T.; Aguirre, G.; André, C.; Bannasch, D.; Becker, D.; Davis, B.; Ekenstedt, K.; Faller, K.; et al. A comprehensive biomedical variant catalogue based on whole genome sequences of 582 dogs and eight wolves. Anim. Genet. 2019, 50, 695-704. [CrossRef] [PubMed]

17. Thorvaldsdóttir, H.; Robinson, J.T.; Mesirov, J.P. Integrative Genomics Viewer (IGV): High-performance genomics data visualization and exploration. Brief. Bioinform. 2013, 14, 178-192. [CrossRef] [PubMed]

18. Rozen, S.; Skaletsky, H. Primer3 on the WWW for General Users and for Biologist Programmers. In Bioinformatics Methods and Protocols; Humana Press: Totowa, NJ, USA, 2000; Volume 132, pp. 365-386. ISBN 978-0-89603-732-8. [CrossRef] 
19. Whitford, W.; Hawkins, I.; Glamuzina, E.; Wilson, F.; Marshall, A.; Ashton, F.; Love, D.R.; Taylor, J.; Hill, R.; Lehnert, K.; et al. Compound heterozygous SLC19A3 mutations further refine the critical promoter region for biotin-thiamine-responsive basal ganglia disease. Mol. Case Stud. 2017, 3, a001909. [CrossRef] [PubMed]

20. Eudy, J.D.; Spiegelstein, O.; Barber, R.C.; Wlodarczyk, B.J.; Talbot, J.; Finnell, R.H. Identification and characterization of the human and mouse SLC19A3 gene: A novel member of the reduced folate family of micronutrient transporter genes. Mol. Genet. Metab. 2000, 71, 581-590. [CrossRef] [PubMed]

21. Reidling, J.C.; Lambrecht, N.; Kassir, M.; Said, H.M. Impaired Intestinal Vitamin B1 (Thiamin) Uptake in Thiamin Transporter-2-Deficient Mice. Gastroenterology 2010, 138, 1802-1809. [CrossRef]

22. Karczewski, K.J.; Francioli, L.C.; Tiao, G.; Cummings, B.B.; Alföldi, J.; Wang, Q.; Collins, R.L.; Laricchia, K.M.; Ganna, A.; Birnbaum, D.P.; et al. The mutational constraint spectrum quantified from variation in 141,456 humans. Nature 2020, 581, 434-443. [CrossRef]

Publisher's Note: MDPI stays neutral with regard to jurisdictional claims in published maps and institutional affiliations.

(C) 2020 by the authors. Licensee MDPI, Basel, Switzerland. This article is an open access article distributed under the terms and conditions of the Creative Commons Attribution (CC BY) license (http://creativecommons.org/licenses/by/4.0/). 